PROCEEDINGS OF THE

AMERICAN MATHEMATICAL SOCIETY

Volume 136, Number 10, October 2008, Pages 3697-3700

S 0002-9939(08)09340-4

Article electronically published on May 7, 2008

\title{
NO EMBEDDINGS OF SOLENOIDS INTO SURFACES
}

\author{
BOJU JIANG, SHICHENG WANG, AND HAO ZHENG \\ (Communicated by Alexander N. Dranishnikov)
}

\begin{abstract}
A quick proof of Bing's theorem indicated by the title is given. Indeed the inverse limit of a sequence of degree $>1$ maps between closed oriented $m$-manifolds can never be embedded into any closed orientable $(m+$ 1)-manifold. The proof also concludes Gumerov's result on the covering of solenoids.
\end{abstract}

In this paper we always assume that a solenoid is not a manifold.

In his two important papers on solenoids, Bing proved first that no solenoid is planar B1 and then if a solenoid can be embedded into surfaces, then it must be planar [B2], and therefore no solenoid can be embedded into surfaces. We will give a short proof of this result. Indeed we show that the inverse limit of a sequence of maps of degree $>1$ between closed oriented $m$-manifolds can never be embedded into any closed orientable $(m+1)$-manifold. The proof also concludes a recent result of $\mathrm{Gu}$ ] on covering degrees of solenoids.

For the sequence $\left\{\phi_{n}: X_{n} \rightarrow X_{n-1}\right\}_{n \geq 1}$, its inverse limit $\lim X_{n}$ is defined as the subspace

$$
\left\{\left(x_{0}, x_{1}, \ldots, x_{n}, \ldots\right) \mid x_{n} \in X_{n}, x_{n-1}=\phi_{n}\left(x_{n}\right)\right\}
$$

of the product space $\prod_{n=0}^{\infty} X_{n}$.

Identify $S^{1}$ with the abelian Lie group $U_{1}=\{z \in \mathbb{C}|| z \mid=1\}$ and let $\phi_{n}: S^{1} \rightarrow$ $S^{1}$ be the homomorphism defined by $z \mapsto z^{w_{n}}$ where $w_{n}>1$ is an integer. For the sequence $\left\{\phi_{n}: S^{1} \rightarrow S^{1}\right\}_{n \geq 1}$, its inverse limit, denoted as $\Theta$, is called the solenoid of type $\varpi=\left(w_{1}, w_{2}, \ldots, w_{n}, \ldots\right)$. By definition $\Theta$ is a connected, closed and hence compact subgroup of the abelian topological group $\prod_{n=0}^{\infty} S^{1}$ (cf. [Mc]).

Theorem 1. (1) For a sequence $\left\{\phi_{n}: X_{n} \rightarrow X_{n-1}\right\}_{n>1}$ of non-zero degree maps of closed oriented $m$-manifolds having infinitely many degree $\phi_{n} \neq \pm 1$, there is no embedding of $\lim X_{n}$ into any closed oriented $(m+1)$-manifolds $M$.

(2) No solenoid $\Theta$, can be embedded into a surface.

Remark 2. In general, we call the inverse limit of maps $\left\{\phi_{n}: X_{n} \rightarrow X_{n-1}\right\}_{n \geq 1}$ an $m$-dimensional solenoid if all $X_{n}$ are closed oriented $m$-manifolds and all $\phi_{n}$ are coverings of degree $>1$. By Theorem 1 1 (1), there is no embedding of $m$-dimensional solenoids into $\mathbb{R}^{m+1}$. This fact has been proved for homogeneous solenoids by Prajs $[\mathrm{Pr}$. Note that solenoids of dimension $>1$ need not be homogeneous unless the compositions of all coverings are regular.

Received by the editors November 2, 2006, and, in revised form, August 9, 2007.

2000 Mathematics Subject Classification. Primary 54F15, 57N35.

The authors were supported by an NSFC grant. 
Theorem 3. If $X$ is a finite-fold covering of a solenoid $\Theta$, then each component of $X$ is homeomorphic to $\Theta$.

Remark 4. According to the description in [Gu, p. 2775], it seems Theorem 3 can be derived from several papers of Fox and Moore in the 1970's, and of Grigorian and Gumerov recently. We will give a direct proof of Theorem 3 .

Proof of Theorem 1. (1) Suppose there is an embedding $\lim X_{n} \subset M$ for some closed orientable $(m+1)$-manifold $M$. We have to seek a contradiction.

According to the continuity of Cech theory, for any commutative ring $R$, the Cech cohomology of the inverse limit is the direct limit of the singular cohomology, that is, $\check{H}^{*}\left(\lim X_{n} ; R\right)=\lim H^{*}\left(X_{n} ; R\right)$. See [ES, Chapter X, Theorems 2.2 and 3.1].

Clearly we have $H^{m}\left(X_{n} ; R\right)=R$. Denote the degree of $\phi_{n}$ by $w_{n}$. Then the Čech cohomology group $\check{H}^{m}\left(\lim X_{n} ; R\right)$ is the direct limit of $R \stackrel{w_{1}}{\longrightarrow} R \stackrel{w_{2}}{\longrightarrow} R \stackrel{w_{3}}{\longrightarrow} R \stackrel{w_{4}}{\longrightarrow}$ $\cdots$. Since all $w_{n} \neq 0$ and infinitely many $w_{n} \neq \pm 1$, it follows that $\check{H}^{m}\left(\underset{\lim }{\longleftarrow} X_{n} ; \mathbb{Z}\right)$ is an infinitely generated $\mathbb{Z}$-module, but $\check{H}^{m}\left(\lim X_{n} ; \mathbb{Q}\right) \cong \mathbb{Q}$ is a finitely generated $\mathbb{Q}$-module.

By the Alexander duality $\check{H}^{m}\left(\lim _{\longleftarrow} X_{n} ; R\right) \cong H_{1}\left(M, M \backslash \underset{\lim }{\longleftarrow} X_{n} ; R\right)$ (cf. GG p. 233]), we have the exact sequence

$$
\cdots \rightarrow H_{1}(M ; R) \rightarrow \check{H}^{m}\left(\lim _{\longleftarrow} X_{n} ; R\right) \rightarrow H_{0}\left(M \backslash \longleftarrow \lim _{\longleftarrow} X_{n} ; R\right) \rightarrow H_{0}(M ; R) \rightarrow \cdots .
$$

Since $H_{*}(M ; R)$ is a finitely generated $R$-module for any commutative ring $R$, the above exact sequence implies that $H_{0}\left(M \backslash \lim X_{n} ; \mathbb{Z}\right)$ is an infinitely generated $\mathbb{Z}$-module and $H_{0}\left(M \backslash \lim X_{n} ; \mathbb{Q}\right)$ is a finitely generated $\mathbb{Q}$-module. But on the other hand $H_{0}\left(M \backslash \lim _{\longleftarrow} X_{n} ; \mathbb{Z}\right)$ is a free abelian group and $H_{0}\left(M \backslash \lim X_{n} ; \mathbb{Q}\right)=$ $H_{0}\left(M \backslash \longleftarrow \lim X_{n} ; \mathbb{Z}\right) \otimes_{\mathbb{Z}} \mathbb{Q}$, so $H_{0}\left(M \backslash \lim _{\longleftarrow} X_{n} ; \mathbb{Z}\right)$ and $H_{0}\left(M \backslash \lim _{\longleftarrow} X_{n} ; \mathbb{Q}\right)$ should have the same basis, a contradiction.

(2) Suppose there is an embedding $\Theta \subset F$ of a solenoid $\Theta$ into a surface $F$. First, we may assume $F$ is a closed surface. If not, since $\Theta$ is compact, $\Theta$ is contained in the interior of some connected, compact subsurface $F^{\prime} \subset F$. Capping a disc on each component of $\partial F^{\prime}$, we get an embedding of $\Theta$ into a closed surface.

According to Theorem 1 (1), $F$ cannot be orientable. If $F$ is not orientable, we consider the orientable double covering $\pi: \tilde{F} \rightarrow F$. Since $\pi^{-1}(\Theta)$ is a double covering of $\Theta$, by Theorem 3 each component of $\pi^{-1}(\Theta)$ is homeomorphic to $\Theta$; hence we get an embedding $\Theta \subset \tilde{F}$ into a closed, orientable surface, which still contradicts Theorem 1 (1).

Proof of Theorem 3. Suppose $\Theta$ is a solenoid of type $\varpi=\left(w_{1}, w_{2}, \ldots, w_{n}, \ldots\right)$ and $\pi: X \rightarrow \Theta$ is an $r$-fold covering.

Let $\Gamma_{n}$ be the kernel of the projection $p_{n}: \Theta \rightarrow S^{1}$ onto the $n$th coordinate, i.e.

$$
\Gamma_{n}=\left\{\left(x_{0}, x_{1}, \ldots, x_{n}, \ldots\right) \in \Theta \mid x_{0}=x_{1}=\cdots=x_{n}=1\right\} .
$$

We have the infinite sequence of closed, and hence compact, subgroups (indeed each $\Gamma_{n}$ is homeomorphic to the Cantor set; cf. $[\mathrm{Mc}$ )

$$
\Theta>\Gamma_{0}>\Gamma_{1}>\Gamma_{2}>\cdots>\Gamma_{n}>\cdots .
$$


The right multiplication of $\Gamma_{n}$ on $\Theta$ makes the projection $p_{n}: \Theta \rightarrow S^{1}$ a principal fiber bundle (cf. [Mc]). It follows that $\Theta$ is the mapping torus of a left transformation $\psi_{n}: \Gamma_{n} \rightarrow \Gamma_{n}$. We choose the left transformation $\psi_{n}$ as follows. Fix a closed path $\alpha(t)=e^{2 \pi i t}, t \in[0,1]$, in $S^{1}$. Then $\alpha_{n}(t)=\gamma\left(w_{1} \cdots w_{n} t\right)$ is a lift of $\alpha$ via the projection $p_{n}$, where

$$
\gamma(t)=\left(e^{2 \pi i t}, e^{2 \pi i t / w_{1}}, e^{2 \pi i t / w_{1} w_{2}}, \ldots, e^{2 \pi i t / w_{1} \cdots w_{n}}, \ldots\right)
$$

is the one-parameter subgroup of $\Theta$. Let $\psi_{n}$ be given by $x \mapsto \alpha_{n}(1) x$.

Moreover, as the covering space of $\Theta, X$ is the mapping torus of a lift $\tilde{\psi}_{n}$ of $\psi_{n}$ which is uniquely determined as follows. For each $\tilde{x} \in \pi^{-1}\left(\Gamma_{n}\right)$, let $\tilde{\alpha}$ be the unique lift starting from $\tilde{x}$ of the path $\alpha_{n}(t) \pi(\tilde{x})$ in $\Theta$ via the covering map $\pi$; then $\tilde{\psi}_{n}(\tilde{x})=\tilde{\alpha}(1)$. Note that $\psi_{n}=\left.\psi_{0}^{w_{1} \cdots w_{n}}\right|_{\Gamma_{n}}$ and hence $\tilde{\psi}_{n}=\left.\tilde{\psi}_{0}^{w_{1} \cdots w_{n}}\right|_{\pi^{-1}\left(\Gamma_{n}\right)}$.

Claim 1. Let $\mathcal{U}$ be an open covering of $\Gamma_{0}$. Then for sufficiently large $n$ each coset of $\Gamma_{n}$ in $\Gamma_{0}$ is contained in some element of $\mathcal{U}$.

Proof. Note that $\mathcal{B}=\left\{x \Gamma_{n} \mid n \geq 0, x \in \Gamma\right\}$ forms a basis for the open sets of $\Gamma_{0}$. So the covering $\mathcal{U}$ has a refinement $\mathcal{U}^{\prime}$ which consists of elements of $\mathcal{B}$. Since $\Gamma_{0}$ is compact we may assume $\mathcal{U}^{\prime}$ is finite. Therefore, for sufficiently large $n$ each coset of $\Gamma_{n}$ in $\Gamma_{0}$ is contained in some element of $\mathcal{U}^{\prime}$, hence in some element of $\mathcal{U}$.

Claim 2. We have $\pi^{-1}\left(\Gamma_{0}\right) \cong \Gamma_{0} \times\{1,2, \ldots, r\}$ as covering spaces of $\Gamma_{0}$.

Proof. Let $\mathcal{U}$ be the open covering of $\Gamma_{0}$ which consists of the open fundamental regions of the covering map $\left.\pi\right|_{\pi^{-1}\left(\Gamma_{0}\right)}$ and let $n$ be sufficiently large as in Claim 1. Then each coset $x \Gamma_{n}$ in $\Gamma_{0}$ is contained in some element $\mathcal{U}$; hence $\pi^{-1}\left(x \Gamma_{n}\right) \cong$ $x \Gamma_{n} \times\{1,2, \ldots, r\}$ as covering spaces of $x \Gamma_{n}$. Since the cosets of $\Gamma_{n}$ are disjoint and open in $\Gamma_{0}$, the claim follows.

In what follows we fix a homeomorphism from Claim 2 and identify both sets.

Claim 3. For each sufficiently large $n$ there exists a permutation $\sigma_{n}$ of $\{1,2, \ldots, r\}$ such that $\tilde{\psi}_{n}(x, j)=\left(\psi_{n}(x), \sigma_{n}(j)\right)$.

Proof. Applying Claim 1 on the the open covering of $\Gamma_{0}$ which consists of the open (and closed) sets $U_{\sigma}=\left\{x \in \Gamma_{0} \mid \tilde{\psi}_{0}(x, j)=\left(\psi_{0}(x), \sigma(j)\right)\right\}$ with $\sigma$ running over all permutations of $\{1,2, \ldots, r\}$, one notices that for sufficiently large $n$ each coset of $\Gamma_{n}$ in $\Gamma_{0}$ is contained in some $U_{\sigma}$. Since $\tilde{\psi}_{n}=\left.\tilde{\psi}_{0}^{w_{1} \cdots w_{n}}\right|_{\pi^{-1}\left(\Gamma_{n}\right)}$, the claim follows.

By Claim $3 X$ is the disjoint union of the mapping tori of $\left.\tilde{\psi}_{n}\right|_{\Gamma_{n} \times J}$ where $J$ runs over all $\sigma_{n}$-orbits. Since $\Theta$ is connected, the following two claims eventually establish the theorem. Below we denote by $T_{f}$ the mapping torus of a self homeomorphism $f$.

Claim 4. For sufficiently large $n$ the length of each $\sigma_{n}$-orbit is relatively prime to $w_{n^{\prime}}$ for all $n^{\prime}>n$.

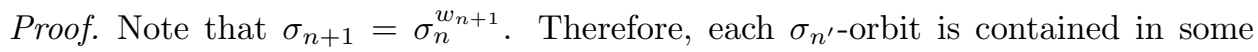
$\sigma_{n}$-orbit for $n^{\prime}>n$ and if the length of a $\sigma_{n}$-orbit $J$ is not relatively prime to $w_{n^{\prime}}$ for some $n^{\prime}>n$, then $J$ splits into several $\sigma_{n^{\prime}}$-orbits. Since a permutation of $\{1,2, \ldots, r\}$ has at most $r$ orbits, the claim follows. 
Claim 5. Let $n$ be sufficiently large as in Claim 3. If the length of a $\sigma_{n}$-orbit $J$ is relatively prime to $w_{n^{\prime}}$ for all $n^{\prime}>n$, then $T_{\left.\tilde{\psi}_{n}\right|_{\Gamma_{n} \times J}} \cong \Theta$.

Proof. Let $l=|J|$ be the length of $J$. It is clear that $T_{\left.\tilde{\psi}_{n}\right|_{\Gamma_{n} \times J}} \cong T_{\psi_{n}^{l}}$. Note that $\Gamma_{n}$ is the inverse limit of the sequence

$$
\left\{\phi_{n+k}: \operatorname{Ker}\left(\phi_{n+1} \cdots \phi_{n+k}\right) \rightarrow \operatorname{Ker}\left(\phi_{n+1} \cdots \phi_{n+k-1}\right)\right\}_{k \geq 1} .
$$

Since $l$ is relatively prime to $w_{n^{\prime}}$ for all $n^{\prime}>n$, the homomorphisms

$$
\operatorname{Ker}\left(\phi_{n+1} \cdots \phi_{n+k}\right) \rightarrow \operatorname{Ker}\left(\phi_{n+1} \cdots \phi_{n+k}\right)
$$

defined by $x \mapsto x^{l}$ are isomorphic. It follows that the homomorphism $\Gamma_{n} \rightarrow \Gamma_{n}$ defined by $x \rightarrow x^{l}$ is isomorphic, via which one notices that $\psi_{n}$ is topologically conjugate to $\psi_{n}^{l}$. So we have $T_{\psi_{n}} \cong T_{\psi_{n}^{l}}$ and therefore, $T_{\left.\tilde{\psi}_{n}\right|_{\Gamma_{n} \times J}} \cong T_{\psi_{n}^{l}} \cong T_{\psi_{n}} \cong$ $\Theta$.

From Claim 4 and Claim 5 we also have

Corollary $5([\mathbf{G u}])$. A solenoid of type $\varpi=\left(w_{1}, w_{2}, \ldots, w_{n}, \ldots\right)$ has a connected $r$-fold covering if and only if $r$ is relatively prime to all but finitely many $w_{n}$.

\section{ACKNOWLEDGEMEnts}

The present form of Theorem 1 is influenced by the referee's suggestion to show no embedding of a (non-torus) $m$-dimensional connected compact Abelian topological group (cf. $[\mathrm{KW}]$ ) into $\mathbb{R}^{m+1}$.

\section{REFERENCES}

[B1] Bing, R. H. A simple closed curve is the only homogeneous bounded plane continuum that contains an arc, Canad. J. Math. 12 (1960), 209-230. MR0111001 (22:1869)

[B2] Bing, R. H. Embedding circle-like continua in the plane, Canad. J. Math. 14 (1962), 113128. MR0131865 (24:A1712)

[ES] Eilenberg, S.; Steenrod, N. Foundations of algebraic topology, Princeton University Press, Princeton, New Jersey, 1952. MR0050886 (14:398b)

[GH] Greenberg, M. J.; Harper, J. R. Algebraic topology. A first course, Mathematics Lecture Note Series 58, Benjamin/Cummings Publishing Co., Inc., Reading, MA, 1981. MR643101 (83b:55001)

[Gu] Gumerov, R. N. On finite-sheeted covering mappings onto solenoids, Proc. Amer. Math. Soc. 133 (2005), 2771-2778. MR2146226 (2006d:54024)

[KW] Keesling, J.; Wilson, D. Embedding $T^{n}$-like continua in Euclidean space. Topology Appl. 21 (1985), no. 3, 241-249. MR812642 (87h:54033)

[Mc] McCord, M. C. Inverse limit sequences with covering maps, Trans. Amer. Math. Soc. 114 (1965), no. 1, 197-209. MR0173237 (30:3450)

[Pr] Prajs, J. Homogeneous continua in Euclidean $(n+1)$-space which contain an $n$-cube are n-manifolds. Trans. Amer. Math. Soc. 318 (1990), no. 1, 143-148. MR943307 (90f:54055)

Department of Mathematics, Peking University, Beijing 100871, People's Republic of CHINA

E-mail address: bjjiang@math.pku.edu.cn

Department of Mathematics, Peking University, Beijing 100871, People's Republic of CHINA

E-mail address: wangsc@math.pku.edu.cn

Department of Mathematics, Zhongshan University, Guangzhou 510275, People's Republic of China

E-mail address: zhenghao@sysu.edu.cn 